\title{
Plasma Enhanced Fluorine-Free Superhydrophobic Polyester (PET) Fabric with Ultra-Robust Antibacterial and Antibacterial Adhesion Properties
}

\author{
Yuling Lai ${ }^{1,2}$, Ying Guo ${ }^{1,3, *}$, Liyun Xu ${ }^{4}$, Xijiang Chang ${ }^{1,3}$, Xingqun Zhang ${ }^{5}$, Guangbiao Xu ${ }^{1,2}$ and Jianjun Shi ${ }^{1,3}$ \\ 1 Shanghai Collaborative Innovation Center for High Performance fiber composites, College of Science, \\ Donghua University, Shanghai 201620, China; hoppings@foxmail.com (Y.L.); changxj@dhu.edu.cn (X.C.); \\ guangbiao_xu@dhu.edu.cn (G.X.); jshi@dhu.edu.cn (J.S.) \\ 2 Innovation Center for Textile Science and Technology, Ministry of Education, Donghua University, \\ Shanghai 201620, China \\ 3 Member of Magnetic Conflnement Fusion Research Center, Textiles Key Laboratory for Advanced Plasma \\ Technology and Application, Donghua University, Shanghai 201620, China \\ 4 College of Textile and Clothing, Nantong University, Nantong 226019, China; gangsheng1989@126.com \\ 5 College of Chemistry, Chemical Engineering and Biotechnology, Donghua University, \\ Shanghai 201620, China; xqz@dhu.edu.cn \\ * Correspondence: guoying@dhu.edu.cn; Tel.: +86-(021)67792090-564
}

Citation: Lai, Y.; Guo, Y.; Xu, L.; Chang, X.; Zhang, X.; Xu, G.; Shi, J. Plasma Enhanced Fluorine-Free Superhydrophobic Polyester (PET) Fabric with Ultra-Robust Antibacterial and Antibacterial Adhesion Properties. Coatings 2021, 11, 15. https://dx.doi.org/10.3390/ coatings11010015

Received: 19 November 2020 Accepted: 22 December 2020

Published: 25 December 2020

Publisher's Note: MDPI stays neutral with regard to jurisdictional claims in published maps and institutional affiliations.

Copyright: (C) 2020 by the authors. Licensee MDPI, Basel, Switzerland. This article is an open access article distributed under the terms and conditions of the Creative Commons Attribution (CC BY) license (https: / / creativecommons.org/ licenses/by/4.0/).

\begin{abstract}
Superhydrophobic antibacterial fabric possesses properties of antibacterial and antibacterial adhesion and shows huge demand in the field of medical textiles. However, current technologies are unable to fully address this. Hence, a simple method is highly desirable. Herein, the pristine polyester (PET) fabric is immersed into the solution containing $\mathrm{ZnO}$ nanoparticle and polydimethylsiloxane (PDMS), and the fiber surfaces are uniformly covered by a ZnO-PDMS layer after being treated by low pressure Ar plasma. The weight gain rate of the treated fabric is $3.5 \%$, which is basically unchanged, and the air permeability, moisture permeability, and tensile properties of the fabric are basically not affected. It is found that the water contact angle (WCA) of the fabric is over $162.7^{\circ}$ and sliding angle (SA) is less than $10^{\circ}$. The stable binding of PDMS and PET fibers induces a robust superhydrophobicity; even after 300 washing cycles and 600 friction cycles, it still remains superhydrophobic. The antibacterial rates of Escherichia coli and Staphylococcus aureus before washing were $99.89 \%$ and $99.85 \%$, respectively, and after 100 cycles of washing, the antibacterial rates decreased to $99.36 \%$ and $99.17 \%$, respectively. Therefore, it shows a good development prospect in the application of protecting clothing or textiles that require good antibacterial properties (such as bed sheets, duvet covers, etc.).
\end{abstract}

Keywords: superhydrophobic; polyester fabrics; fluorine-free; antibacterial; antibacterial adhesion

\section{Introduction}

Textiles are widely used in clothing, household items, and other fields because of their excellent tensile strength, tailorability, skin-fitting, and other performance. Textile has a unique pore structure that makes it breathable, but if in contact with human skin, this unique structure easily retains sweat, sebum, and other excreta. Moreover, some textiles like cotton, wool, and silk are nutrients, so textiles can often provide a foothold and food for the growth of microorganisms, which makes it easy for microorganisms to adhere and grow on the fabric surface. For example, bacterial adhesion could form a biofilm on the surface of the material, which will cause more bacteria to adhere. The existence of bacteria poses a great potential harm to human health. Mild cases can stimulate the skin and then cause skin diseases. In severe cases, they can invade the immune system and even endanger life. 
Generally, bacteria are adsorbed on the surface of materials by van der Waals force and electrostatic attraction. Then, the bacteria secreted mucus and capsular polysaccharide, and further improved the adhesion to the material surface through the flagellum and pili. Finally, the first batch of bacteria attached to the surface of the material began to propagate continuously, and the biofilm formed by mucus and capsular polysaccharide provided new sites for other free bacteria. So, inhibition of the adhesion of bacteria at the initial stage is an effective means to eliminate the adhesion of bacteria on the material surface [1]. The factors affecting bacterial adhesion mainly include the species of bacteria, surface energy, surface microstructure, hydrophilic hydrophobic balance, and so on [2]. Through further research, scholars have developed a variety of different antibacterial adhesion surfaces, including superhydrophobic anti-adhesion surface, hydrophilic anti-adhesion surface, underwater super oil repellent anti-adhesion surface, and intelligent antibacterial anti-adhesion surface [3]. Among them, the superhydrophobic anti-adhesion surface has received widespread attention for its huge application potential. Stallard et al. [4] pointed out that the superhydrophobic surface has good antibacterial properties and can significantly reduce the adsorption of protein. Nguyen-Tri found out that the reason why superhydrophobic surfaces can prevent bacterial adhesion is that the microstructure with nano-surface can capture a stable air cushion, which makes it difficult for microorganisms to approach and adhere to the surface of superhydrophobic materials [5]. In addition, water droplets easily roll off the superhydrophobic surface and can take away pollutants such as microorganisms so as to further prevent the microorganism from adhering to the surface [6]. In order to prove this conclusion, Qian et al. [7] studied the properties of superhydrophobic polyester (PET) sheets treated with 2,3,5,6-tetrafluoro-p-phenylenediol (TFPDM), and found that the material could effectively prevent bacterial adhesion and the formation of subsequent biofilm. However, Ellinas [8] pointed out that although the superhydrophobic surface can achieve the antibacterial adhesion function by reducing the bacterial adhesion to a certain extent. Once the bacterial concentration exceeds a certain threshold, the function will be affected. In order to solve this problem, further research has been carried out, and it is found that the superhydrophobic surface can have double synergism of bactericidal and bacterial adhesion. For example, for the Silver ion immobilized superhydrophobic coating prepared by Liu et al. [9], the release of silver particles is delayed due to the existence of air cushion on the superhydrophobic surface, and the antibacterial performance was significantly improved. In addition, the stability and durability of the antibacterial property of the material are further improved. The mechanism is that even when microorganisms are successfully adhered to the surface of the material, the antibacterial agent in the material would kill and decompose the bacteria. Moreover, the bacterial corpse adheres to the surface of the fabric only with difficulty. In this way, the surface of superhydrophobic antibacterial fabric can retain antibacterial properties.

However, the durability of most superhydrophobic and antibacterial fabrics is poor, especially for fabrics with nanoparticles and other inorganic antibacterial agents. This is because mechanical contact would damage the micro roughness characteristics of superhydrophobic surface and superhydrophobic coatings [10]. Hence, when subjected to mechanical damage such as physical friction, the superhydrophobic property of the fabric would be permanently lost, and the inorganic antibacterial agent easily falls off from the fabric so the antibacterial property disappears easily. In order to solve this problem, many research groups have carried out research focusing on improving the mechanical stability of fabrics by strengthening the adhesion between fibers and low surface energy coatings and nanoparticles. The most typical example is $\mathrm{Wu}$ et al. [11], who proposed a solution impregnation method of a three-step continuous deposition of branched chain polyethylenimine (PEI), Ag nanoparticles, and fluorinated decane polyhedral oligomeric silsesquioxane (F-POSS) to deposit composite films on cotton fabrics to obtain superhydrophobic antibacterial fabrics. On the one hand, PEI can connect with the hydroxyl group on cotton fiber through the interaction of hydroxyl and electrostatic. On the other hand, it can connect with AgNPs through the interaction of primary, secondary, and tertiary 
amino groups, so as to improve the firmness of AgNPs. Moreover, Xu et al. [12] deposited zinc oxide nanoparticles and microrods on cellulose by a one-step hydrothermal method. Also, after stearic acid modification, the cellulose film has superhydrophobicity. However, these processes often use higher temperatures [13], longer reaction times [14], complex processes, or multiple steps [15,16], and even use fluorine-containing products [17], which makes it difficult to apply in industrial production [18].

Herein, a simple and rapid method is proposed. The PET fabric is treated for $15 \mathrm{~s}$ by plasma technology after simple solution impregnation at room temperature. This process uses fluorine-free polydimethylsiloxane (PDMS) and zinc oxide nanoparticles, and the whole process is energy-saving and environmentally friendly. The obtained fabric is superhydrophobics and after 600 friction cycles and 300 washing cycles, it still remains superhydrophobic, showing excellent mechanical stability. In addition, the obtained fabric has dual synergistic properties of antibacterial adhesion and antibacterial, and after 100 washing cycles, it still remains strong antibacterial property. The obtained fabric shows huge application prospects in the field of medical textiles (such as medical protective clothing, bed sheet, quilt cover, etc.).

\section{Experiments}

\subsection{Materials and Devices}

Commercially available polyester fabric (grammageis $427 \mathrm{~g} / \mathrm{m}^{2}$ and $100 \%$ polyester) was purchased from a local store and cleaned with acetone and deionized water sequentially in an ultrasonic cleaner before use. Absolute ethanol $(\geq 99.7 \%)$ was purchased from Changzhou Hongsheng Fine Chemical Co., Ltd. (Shanghai, China). Nano zinc oxide particles (M.W. 81.39, $90 \pm 10 \mathrm{~nm}$ ) were obtained from Macklin. All the chemicals were analytical grade reagents and used as received.

Polydimethylsiloxane (PDMS, M.W. 770, CAS: 9016-00-6, $\left.\left(\mathrm{C}_{2} \mathrm{H}_{6} \mathrm{OSi}\right)_{n}\right)$ was purchased from Afaisa (China) Chemical Co., Ltd. (Shanghai, China). It is a kind of flexible silicone monomer with good performance, and has the properties of non-toxic, tasteless, stable physical and chemical, wear resistance, and good elasticity. In addition, the structure of PDMS is relatively simple, and can combine with fiber macromolecules to form an elastic membrane on the surface of fabric, which is suitable for post-treatment of different fibers.

The low-temperature plasma device has been described in the previous article [19]. The plasma was generated by a RF power supplywith13.56 MHz (AE Cesar 1320 Advanced Energy Industries, Inc., Denver, CO, USA). The working gas was Argon and the pressure in the reaction chamber was $50 \mathrm{~Pa}$ during plasma processing. The dipped fabrics were then modified by the plasma for $15 \mathrm{~s}$ with the RF power of $50 \mathrm{~W}$.

\subsection{Preparation of Superhydrophobic and Antibacterial Fabric}

$0.05 \mathrm{~g} \mathrm{ZnO}$ was dispersed in $100 \mathrm{~mL}$ ethanol by stirring in the magnetic stirrer, and then the mixture was transferred to ultrasonic cleaner for further dispersed. $3.5 \mathrm{~g}$ PDMS was added into the above-mixed solution and vibrated continuously for $10 \mathrm{~min}$ to get a homogeneous solution. The cleaned polyester fabric was immersed in the above solution at ambient temperature and then heated in an oven at $70{ }^{\circ} \mathrm{C}$ for $10 \mathrm{~min}$. Finally, the polyester fabric was treated by the plasma (Ar gas, power $50 \mathrm{~W}$, pressure $50 \mathrm{~Pa}$, treating $15 \mathrm{~s})$.

\subsection{Characterizations}

Water repellency of samples was characterized by water contact angle (WCA, DropMeter $^{\text {TM }}$ Professional A-200, Haishu Maishi Testing Technology Co., Ltd., Ningbo, China) and sliding angle (SA). Surface morphology of the fabrics was measured by scanning electron microscope (SEM, Hitachi S-4800, Hitachi, Japan). Energy dispersive spectrometry (EDX) analysis was conducted on X-ray micro analysis system attached to the SEM. Functionality of the surfaces was determined using a Fourier transform infrared spectroscopy (FTIR, Nicolet 6700, Thermo Electron Corporation, Waltham, USA) in the wavelength range 
of $600-4000 \mathrm{~cm}^{-1}$, and the spectrum was processed by omnic software to get a specific analysis result. The washing durability was tested according to GB/T 3921-2008 using a washing fastness tester (SW-8, Fangyuan Instrument Co., Ltd., Wenzhou, China) by washing in $40{ }^{\circ} \mathrm{C}$ water (30 min for one cycle). The abrasion durability test was conducted according to GB/T 3920-2008 by dye rubbing fastness machine (Y571N, Hongda, Nantong, China). During the test, the pressure of $44.8 \mathrm{kPa}$ was employed. At regular intervals of washing or rubbing, WCAs were measured to observe the changes in wettability.

\subsection{Antimicrobial Test}

Agar plate diffusion method (GB/T 20944.1-2007 [20]) and oscillation method (GB/T 20944.3-2008 [21]) were used to test the antibacterial properties of the coated fabrics. Escherichia coli (E. coli, ATCC 10536, Gram-negative) and Staphylococcus aureus (S. aureus, ATCC 6538, Gram-positive) were selected in this test.

Agar plate diffusion method: Escherichia coli and Staphylococcus aureus were activated and was diluted to $1 \times 10^{8}-5 \times 10^{8} \mathrm{CFU} \mathrm{mL}{ }^{-1}$ with sterile distilled water to prepare the inoculation liquid. Take $10 \mu \mathrm{L}$ of the bacterial solution, evenly spread it on the agar surface with a coater. Then place the fabric in the center of the agar plate. After that, the culture dish was placed in $37^{\circ} \mathrm{C}$ incubator for $18-24 \mathrm{~h}$. Then, observe and measure the diameter of the antibacterial zone at least 3 times for each sample, and calculate the antibacterial bandwidth of the sample according to Equation (1). The experimental results were repeated three times and the average value was taken. Where: $\mathrm{H}$ is the width of the antibacterial zone, in $\mathrm{mm}$; $D$ is the average value of the external diameter of the antibacterial zone, in $\mathrm{mm} ; d$ is the diameter of the sample, in $\mathrm{mm}$.

$$
H=(D-d) / 2
$$

Oscillation method: Escherichia coli and Staphylococcus aureus were activated and was diluted to the concentration of $3 \times 10^{5}-4 \times 10^{5}\left(\mathrm{CFU} \mathrm{mL} \mathrm{mL}^{-1}\right.$ ) to prepare the inoculation liquid. Take $5 \mathrm{~mL}$ inoculation solution and $70 \mathrm{~mL} 0.03 \mathrm{~mol} / \mathrm{L}$ PBS (phosphate) buffer solution into a conical flask, and added $0.75 \pm 0.05 \mathrm{~g}$ fabric sample. Then the culture was carried out at a constant temperature of $150^{\circ} \mathrm{R} / \mathrm{min}$ in a conical flask at a constant temperature of $18^{\circ} \mathrm{R} / \mathrm{min}$. After that, $1 \mathrm{~mL}$ of test solution was drawn from each conical flask and added to the test tube containing $9 \mathrm{~mL}$ PBS, shaken, and diluted in series by ten-fold dilution method. Then, $1 \mathrm{~mL}$ bacterial solution was taken from each diluted test tube and added to the sterilized nutrient agar medium. The bacterial suspension was evenly distributed on the agar surface with a coating rod and cultured at $37{ }^{\circ} \mathrm{C}$ for 24-48 $\mathrm{h}$. Then, count the colonies and calculate the antibacterial rate according to the following Equation (2). Where $Y$ is the antibacterial rate of the sample; $W_{\mathrm{t}}$ is the average value of viable bacterial concentration of the control sample in the flask after shaking $18 \mathrm{~h}\left(\mathrm{CFU} \mathrm{mL} \mathrm{m}^{-1}\right) ; Q_{\mathrm{t}}$ is the average concentration of viable bacteria of antibacterial fabric samples in the flask after $18 \mathrm{~h}$ of $\left(\mathrm{CFU} \mathrm{mL}{ }^{-1}\right)$.

$$
Y=\frac{W_{t}-Q_{t}}{W_{t}} \times 100 \%
$$

\subsection{Antibacterial Adhesion Test}

The antibacterial adhesion rate of fabrics can be measured by referring [22]. That is, the pristine and the coated fabric were vertically immersed in a $20 \mathrm{~mL} 10^{5} \mathrm{CFU} \mathrm{mL}{ }^{-1}$ bacterial suspension (E. coli, or S. aureus) and cultured at $37^{\circ} \mathrm{C}$ for $18 \mathrm{~h}$. Before taking out the fabric, keep it vertically for 3 min to make the remaining droplets slide away. Then put the sample into the test tube with PBS in a constant temperature shaker at $37^{\circ} \mathrm{C}$ for $30 \mathrm{~min}$, so that the bacteria adhered on the surface of the sample would fall off. Then $1 \mathrm{~mL}$ of the test solution was drawn from each conical flask, and was added to the test tube containing $9 \mathrm{~mL}$ PBS, and diluted in series by ten-fold dilution method. Take $10 \mathrm{uL}$ of bacterial liquid from the test tube with appropriate dilution ratio by gun head, add it to sterilized nutrient 
agar medium, and make the bacterial suspension evenly distributed on the agar surface with coating rod. After $24 \sim 48 \mathrm{~h}$ cultivation at $37^{\circ} \mathrm{C}$, record the number of colonies, take photos, and calculate the adhesion rate of bacteriostatic bacteria according to the following Equation (3).

$$
Y=\frac{W_{\mathrm{t}}-Q_{\mathrm{t}}}{W_{\mathrm{t}}} \times 100 \%
$$

In the formula, $Y$ is the antibacterial adhesion rate of the sample; $W_{\mathrm{t}}$ is the average value of the bacteria concentration of three control groups $\left(\mathrm{CFU} \mathrm{mL} \mathrm{L}^{-1}\right) ; Q_{\mathrm{t}}$ is the average value of the bacteria concentration of three experience groups $\left(\mathrm{CFU} \mathrm{mL}{ }^{-1}\right)$.

\section{Results and Discussion}

\subsection{Wettability, Surface Morphology, and Chemistry Analysis}

The contact angle measurement technique and SEM were used to observe the wettability behavior and the change of surface morphology of polyester fabric before and after treated. As shown in Figure 1a, the pristine PET fiber has a smooth surface and almost has no impurities, and it is hydrophilic. Figure $1 \mathrm{~b}$ shows the surface morphology of PET fabric after impregnation with PDMS coating and treated by plasma (PET-PDMS fabric), it can be seen that the surface morphology of PET-PDMS fabric is covered with a layer of uniform and compact dense film, and the film is basically complete, proving the PDMS is applied to the fabric. The PET-PDMS fabric is found to have superhydrophobic with WCA $158.6^{\circ}$, and a water droplet can easily roll off with the slide angle (SA) of $8.5^{\circ}$, indicating its superhydrophobic property. Figure 1c shows the surface morphology of fabric that impregnating with PDMS@ZnO coating and treated by Ar plasma (PET-PDMS@ZnO fabric), it can be seen that $\mathrm{ZnO}$ nanoparticles are evenly dispersed on the surface of polyester fiber, and the surface roughness of the fiber is greatly improved. It displays outstanding water repellency with a WCA of $162.7^{\circ}$ and SA of $7.5^{\circ}$ respectively, shows a better superhydrophobic property than PET-PDMS fabric. That is because $\mathrm{ZnO}$ nanoparticles can improve the roughness of the fiber surface. Additionally, other liquids like tea, honey, and hydrogen peroxide, etc., are also superhydrophobic with WCA more than $150^{\circ}$, revealing superhydrophobic property of PET-PDMS@ZnO fabric (Figure 2).

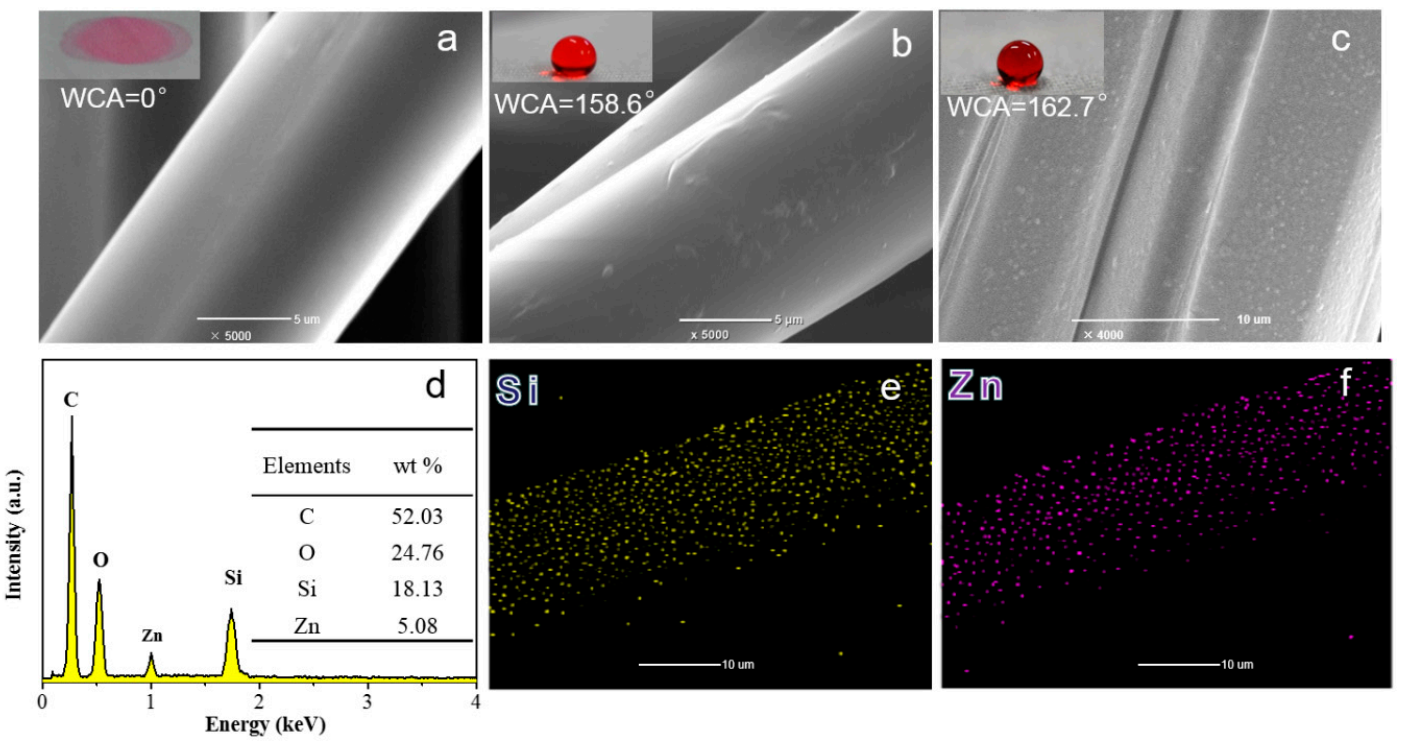

Figure 1. Surface texture and surface chemistry element analysis. SEM images: (a) the pristine farbic, (b) polyesterpolydimethylsiloxane (PET-PDMS) fabric, (c) PET-PDMS@ZnO fabric; (d) EDX spectrum of the PET-PDMS@ZnO fabric; elemental mapping of silicon (e) and zinc (f) for the PET-PDMS@ZnO fabric. 


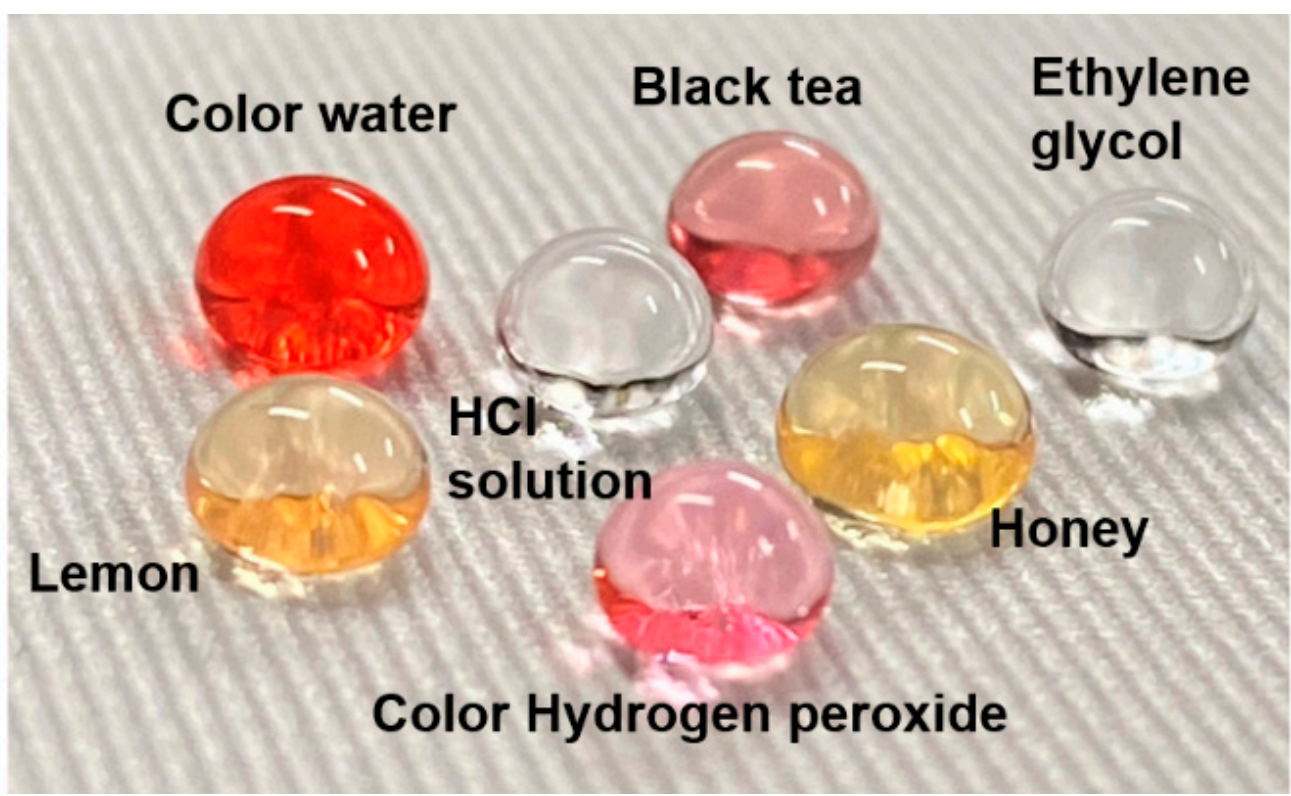

Figure 2. Optical image of PET-PDMS@ZnO fabric consisting of various droplets.

To confirm the existence of silicon and zinc and observe their distribution on polyester fiber, energy dispersive spectrometry (EDX) was used, and the results are shown in Figure 1d-f. As shown in Figure 1d, the rough coating layer is composed of elements of carbon, oxygen, silicon, and zinc. Among them, silicon and zinc are accounting for $18.13 \%$ and $5.08 \%$ respectively. Silicon was derived from PDMS, and zinc was from $\mathrm{ZnO}$. Figure 1e,f show the elemental mapping of silicon and zinc on the fabric surface. It can be seen that the distribution of silicon and zinc is uniform and is consistent with the fiber trend, and there is no obvious accumulation and blank load. The result demonstrates that the PDMS@ZnO layer is uniformly covered on the fiber.

To confirm the presence of a functional group, FTIR was employed and the results are shown Figure 3. Compared with the pristine sample, some characteristic peaks of the PETPDMS@ZnO fabric have changed in intensity and width, the decrease of the characteristic peaks at $842.63 \mathrm{~cm}^{-1}$ and $1252.82 \mathrm{~cm}^{-1}$ corresponds to the symmetric stretching vibration of $\mathrm{Si}-\left(\mathrm{CH}_{3}\right)_{2}$ and $\mathrm{Si}-\left(\mathrm{CH}_{3}\right)_{3}$, the vibration of the chemical bond is conducive to the chemical cross-linking reaction of PDMS during the plasma treatment [23]. The decrease of the peak value in the region of $1100-1030 \mathrm{~cm}^{-1}$ is attributed to the stretching vibration of the $\mathrm{Si}-\mathrm{O}-\mathrm{Si}$ bond [24]. In addition, the decrease of the peak at about $800 \mathrm{~cm}^{-1}$ corresponds to the stretching of the $\mathrm{Si}-\mathrm{OH}$ bond, which indicates that the PDMS molecule has self-condensed, or chemical bonds and chemical cross-linking reactions have occurred with the ester group that formed by the hydrolysis of the polyester macromolecule. These functional groups indicate the long chain alkyl groups and $\mathrm{Si}-\mathrm{O}-\mathrm{Si}$ network structure are formed. The long chain alkyl group is a low surface energy substance, and the Si-O-Si network structure is outstanding stable, which makes the PET-PDMS@ZnO fabric possess superhydrophobic properties and excellent mechanical stability. 


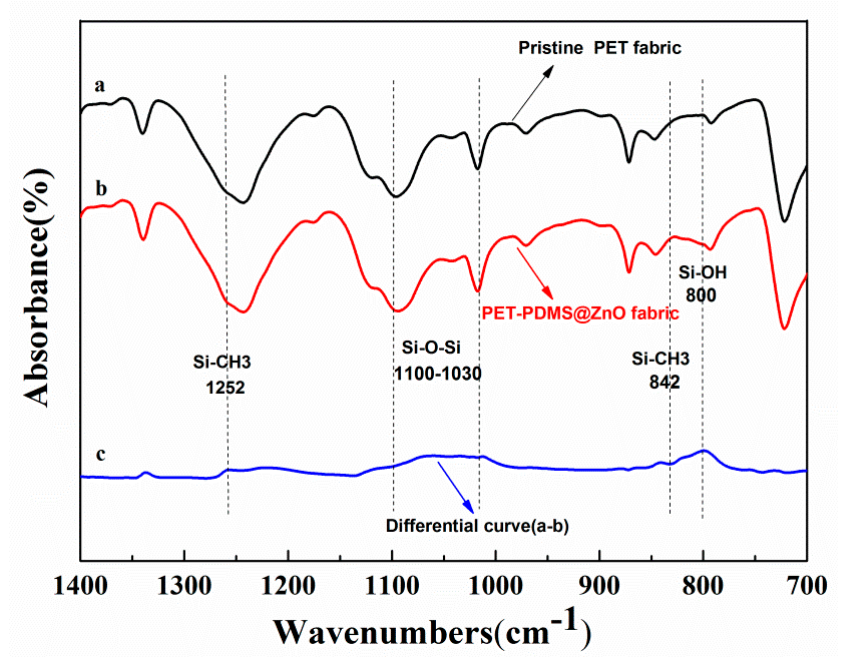

Figure 3. FTIR spectra of samples.

\subsection{Durability of the PET-PDMS@ZnO Fabric}

Mechanical robustness of the coated fabric plays a vital role on its widespread application, as the surface roughness is usually at the micro or nano scale, which is mechanically weak and readily abraded [10]. The durability after washing and abrasion were evaluated. Figure 4a shows the schematic diagram of abrasion test. As the SEM image (Figure 4c) shows, after 600 cycles of abrasion, PDMS@ZnO layer is partially worn off, but still adhered to the fiber surface. Also, it can be seen from Figure $4 \mathrm{~b}$ that after 600 cycles of abrasion, WCA of the fabric decreased from $162.7^{\circ}$ to $145.9^{\circ}$, and SA of the fabric decreased from $7.5^{\circ}$ to $37.5^{\circ}$. After 300 cycles standard washing, WCA and SA decreased to $150.1^{\circ}$ and $30.6^{\circ}$ respectively (as shown in Figure $4 \mathrm{~d}$,e), and the fabric still remained hydrophobic. SEM image shows that there is still PDMS@ZnO coating adhering to the fiber surface after 300 cycles of washing (Figure 4f). This indicates that PDMS@ZnO coating has a strong binding force on the fiber surface, and the coated fabric has excellent friction and washing resistance, which indirectly implies the bonding reaction between PDMS and polyester.

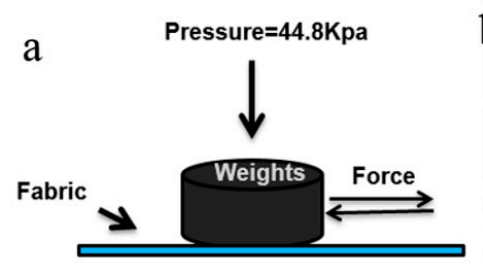

b
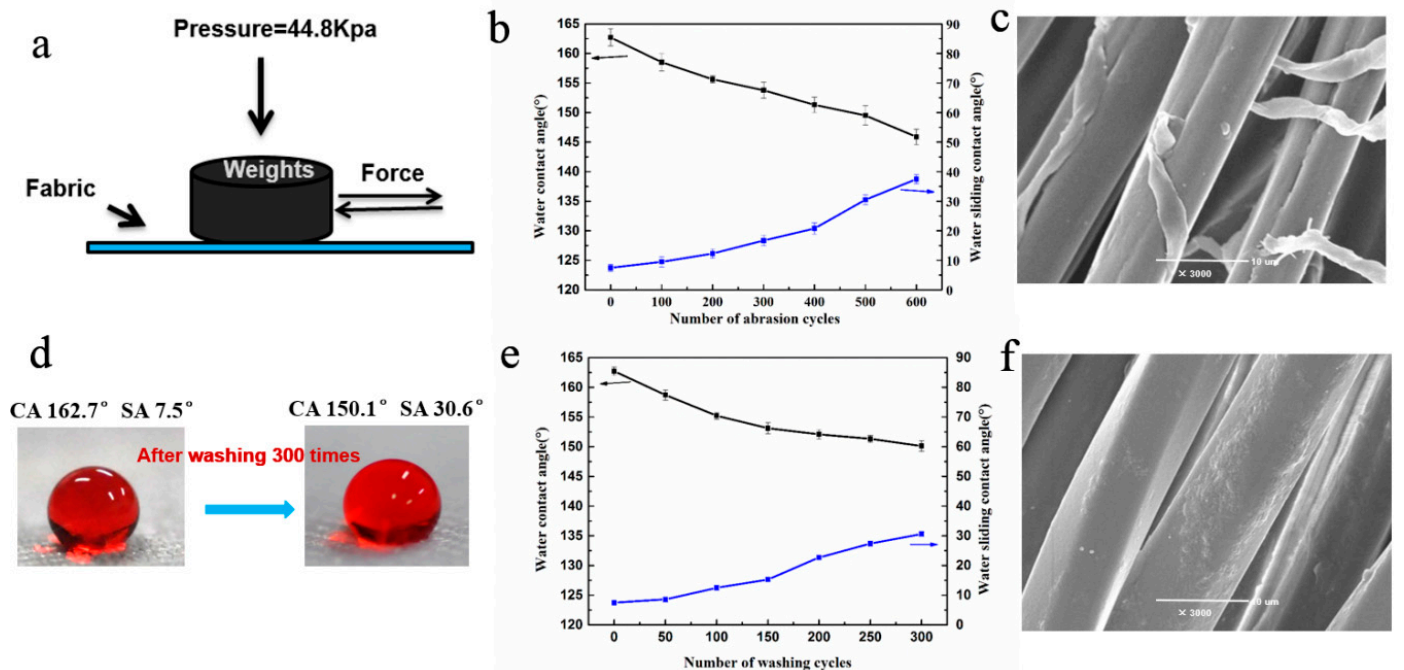

Figure 4. Abrasion and washing tests. (a-c) Abrasion tests: (a) Schematic diagram of abrasion test, (b) Variation of water contact angle and sliding angle with the numbers of abrasion tests, (c) SEM images of the PDMS@ZnO fiber after 600 cycles of abrasion; (d-f) Washing tests: (d) Photos of water drops on the PET-PDMS@ZnO fabric after 300 cycles of washing, (e) Variation of water contact angle and sliding angle with the numbers of washing tests, (c) SEM images of the PDMS@ZnO fiber after 300 cycles of washing. 


\subsection{Antibacterial Property}

Antibacterial property refers to the ability of materials or compounds to inhibit or kill microorganisms so that they can not further grow and reproduce. Antibacterial agents have bactericidal specificity so they do not cause any harmful effects on living tissues [25]. In this paper, the antibacterial activity was tested against the E. coli bacteria (Gram-negative bacteria) and S. aureus (Gram-positive bacteria) by using qualitative measurement (inhabitation zone method) and quantitative measurement (perturbation method). As shown in Figure 5a, no inhibition zone is observed on the pristine fabric and PET-PDMS fabric, while the inhibition zone (no growth of bacteria) surrounding the PET-PDMS@ZnO fabric is observed, and the diameters of inhibition zone for E. coli and S. aureus are approximately 2.57 and $2.16 \mathrm{~mm}$, respectively. After washing 100 times, the diameters of inhibition zone for E. coli and S. aureus on PET-PDMS@ZnO fabric remains 1.98 and $1.75 \mathrm{~mm}$ respectively, revealing the excellent robustness of the antibacterial characteristics of PET-PDMS@ZnO fabric.

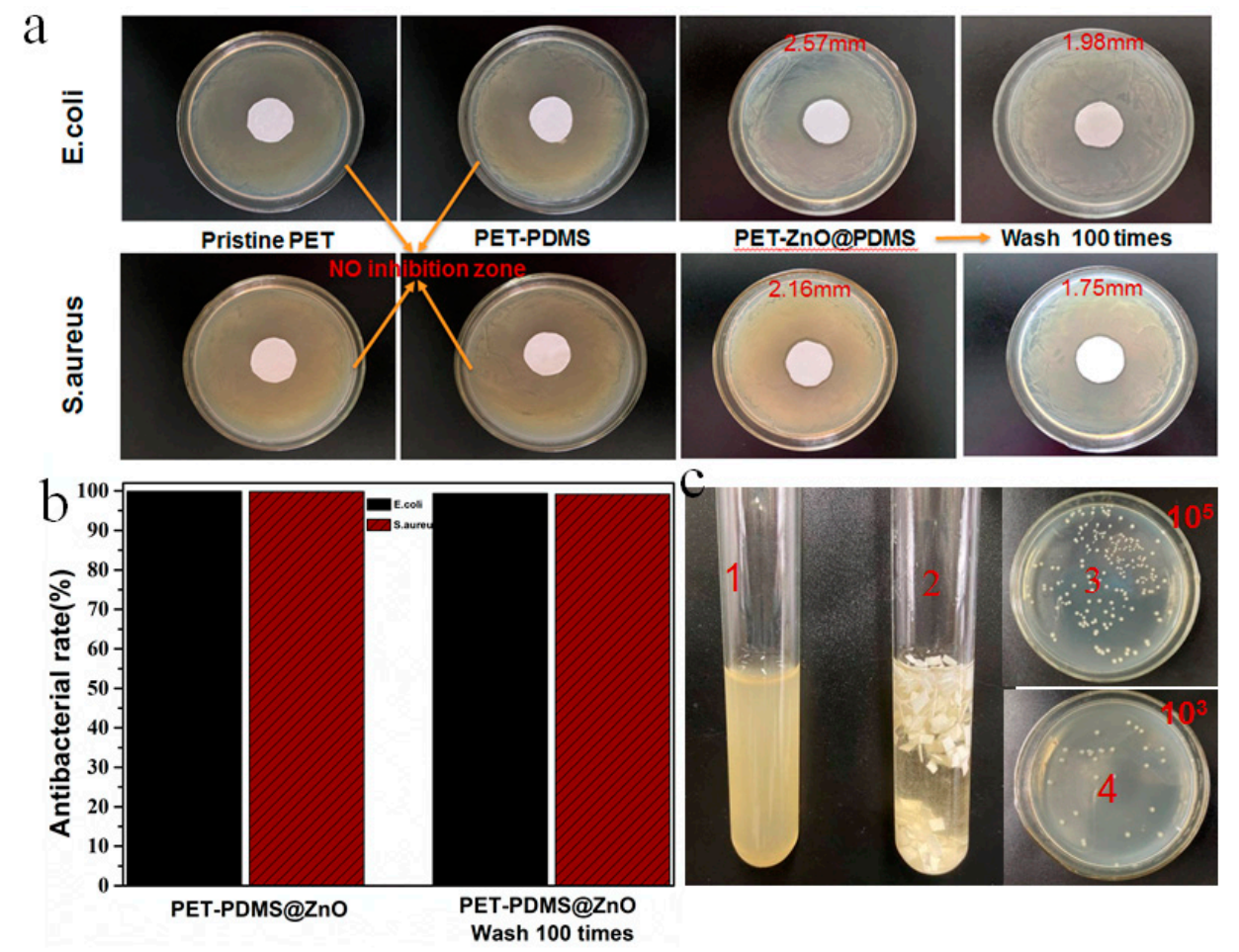

Figure 5. Antimicrobial tests. (a) Optical images of inhibition zone of different fabrics; (b) Bacteriostatic rate of PDMS@ZnO coated fabric before and after washing 100 times, with E. coli and S. aureus bacteria; (c) Blank group (No.1 and 3) and experimental group (sample of PET-PDMS@ZnO, No.2 and 4) of Antimicrobial tests.

Figure $5 \mathrm{~b}$ shows the results of antibacterial activity of PET-PDMS@ZnO fabrics before and after 100 washes by the oscillation method. The antibacterial rates of Escherichia coli and Staphylococcus aureus before washing were $99.89 \%$ and $99.85 \%$, respectively. After 100 cycles of washing, the antibacterial rates decreased to $99.36 \%$ and $99.17 \%$, respectively, showing good antibacterial activities of PET-PDMS@ZnO fabric. Figure $5 \mathrm{c}$ contains blank group (No.1 and 3, standard cotton fabric) and experimental group (No.2 and 4, PET-PDMS@ZnO fabric). The test tube is filled with liquid culture medium, and the same concentration of bacteria liquid is added before the experiment. The results showed that the liquid medium in the blank group (Figure 5c No.1, standard cotton fabric) was turbid, indicating that there were a lot of bacteria in the blank test tube, while the liquid medium in the experimental group (Figure 5c No.2, PET-PDMS@ZnO fabric) was relatively clear, which indicated the bacteria in the experimental group had been basically killed, implying PET-PDMS@ZnO 
fabric has good bactericidal property Figure $5 c-3$ (the blank group) and Figure $5 c-4$ (the experimental group) correspond to the number of bacteria after diluting $10^{5}$ times and $10^{3}$ times respectively, the large bacteria population gap also indicating PET-PDMS@ZnO fabric has strong bactericidal property. This is basically because $\mathrm{ZnO}$ would slowly release $\mathrm{Zn}^{2+}$ in aqueous medium, and $\mathrm{Zn}^{2+}$ could enter the cell through the cell membrane and react with some groups on the protein to destroy the cell structure and physiological activity. Moreover, $\mathrm{Zn}^{2+}$ could sneak into the bacteria to destroy the enzyme of electron transfer system and react with $-\mathrm{SH}$. Then, the objective of sterilization is achieved [26].

\subsection{Antibacterial Adhesion Property}

In this paper, antibacterial adhesion test was conducted. The nano or micron rough structure of superhydrophobic surface can capture the stable air cushion to form a uniform and stable air shield on the surface of the fabric. This makes it difficult for water molecules and microorganisms to get close to and adhere to the surface [5]. The existence of the air shield on the rough structure also makes the fabric hanging on the liquid surface. (Figure 6b). Figure 6a shows the result of antibacterial adhesion test. Standard cotton fabrics were used as a control group. The antibacterial adhesion rate against $E$. coli and S. aureus of pristine PET fabric is $84.33 \%, 82.93 \%$ of PET-PDMS fabric is $90.19 \%, 89.18 \%$ and of PET-PDMS@ZnO fabric is $99.41 \%, 97.71 \%$ respectively. Compared with the standard cotton fabric, the pristine PET fabric has a better antibacterial adhesion rate, which is mainly due to the different fabric structure. In addition, PET fiber is smoother than cotton fiber, which can hinder the adhesion of bacteria to a certain extent. Moreover, natural cotton served as food for bacterial growth, which is more likely to breed pathogenic bacteria than PET fiber [27]. Compared with the PET-PDMS fabric, the antibacterial adhesion rate of PET-PDMS@ZnO fabric is increased by nearly 10\%. As shown in Figure 7, the reason is because the PDMS is coated on the outer layer of $\mathrm{ZnO}$ nanoparticles, and the treatment of plasma induced cross-linking and formed the long-chain alkyl and S-O-Si network structure on the PDMS film, which enhanced the stability of the PDMS@ZnO coating film. On the one hand, the addition of $\mathrm{ZnO}$ nanoparticles improves the surface roughness of the coating and enhances the superhydrophobic property. The superhydrophobic surface of PET-PDMS@ZnO fabric served as the first line of defense to prevent bacteria from invading the fabric surface due to its antibacterial adhesion property. On the other hand, $\mathrm{Zn}^{2+}$ released by $\mathrm{ZnO}$ could interact with some groups of proteins on the cell membrane, destroy the structure and physiological activity of bacteria, and $\mathrm{Zn}^{2+}$ can also enter the interior of bacteria to destroy enzymes, finally leading to bacteria death [26]. So, even if a small amount of bacteria are successfully adhered to the fabric surface, they will be killed and decomposed by zinc oxide nanoparticles contained in the coating. Furthermore, the bacterial corpse is also difficult to adhere to the fabric surface. In this way, the surface of superhydrophobic antibacterial fabric would retain antibacterial properties, and the fabric have double synergism of sterilization and bacterial adhesion [9].

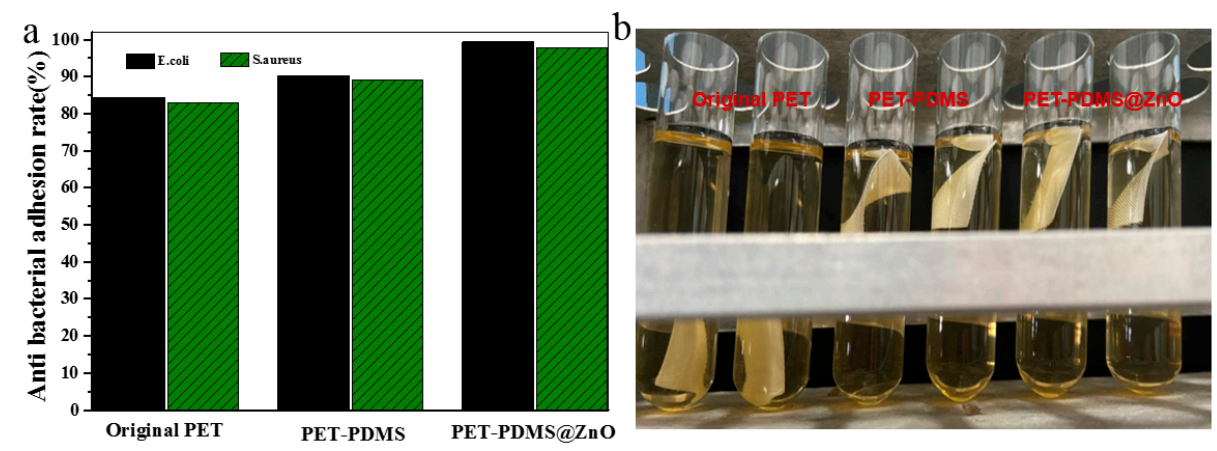

Figure 6. Antibacterial adhesion tests. (a) Antibacterial adhesion rate of different fabric, with E. coli and S. aureus bacteria; (b) The state of different PET fabrics in liquid culture medium. 


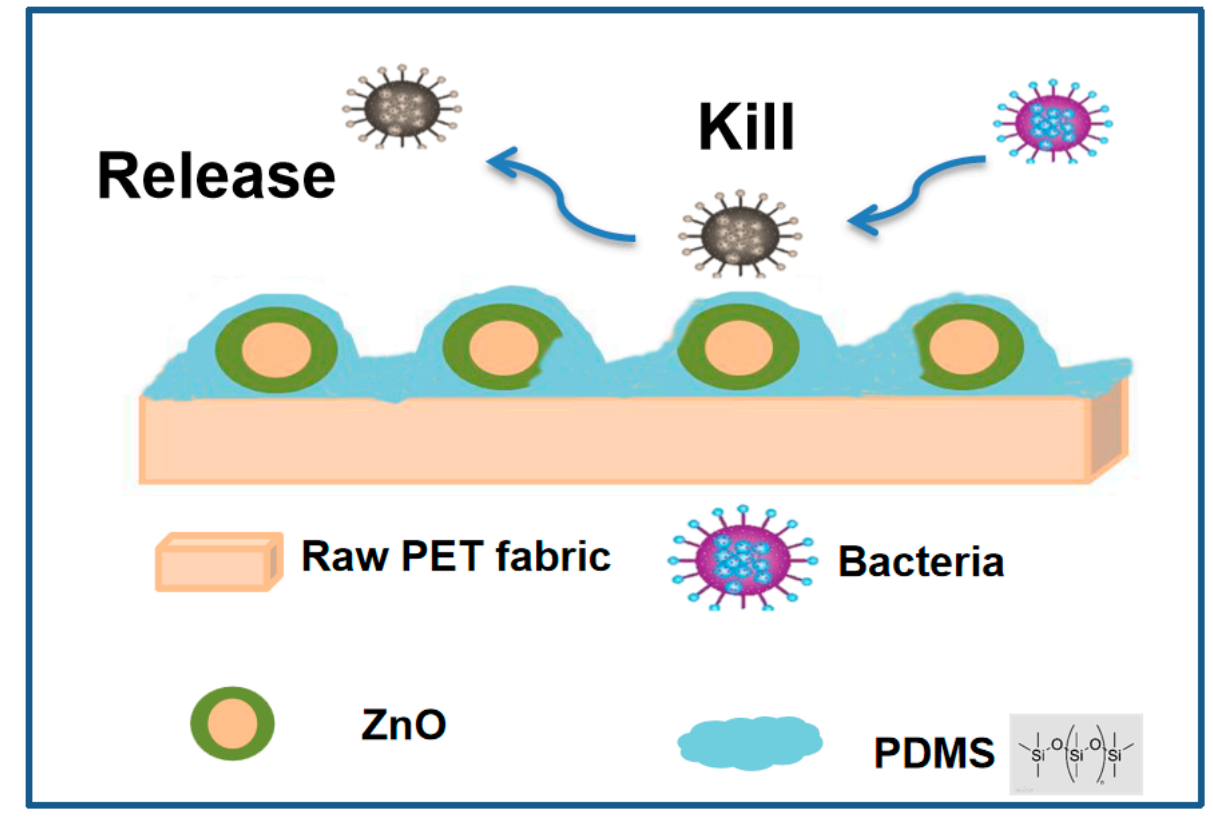

Figure 7. The schematic diagram of synergistic effect of antibacterial and antibacterial adhesion of PET-PDMS@ZnO fabrics.

\subsection{Applications of the PET-PDMS@ZnO Fabric}

In our daily life or in industrial production, the splashing of juice, coffee, or harmful chemical liquids will bring certain influence or harm to people. As shown in Figure 8, water flow diffused or even penetrated into the pristine PET fabric, but was ejected on the PET-PDMS@ZnO fabric, and leaving no trace on the surface of the fabric. It suggested the PET-PDMS@ZnO fabric has strong water repellency and water splashing performance.

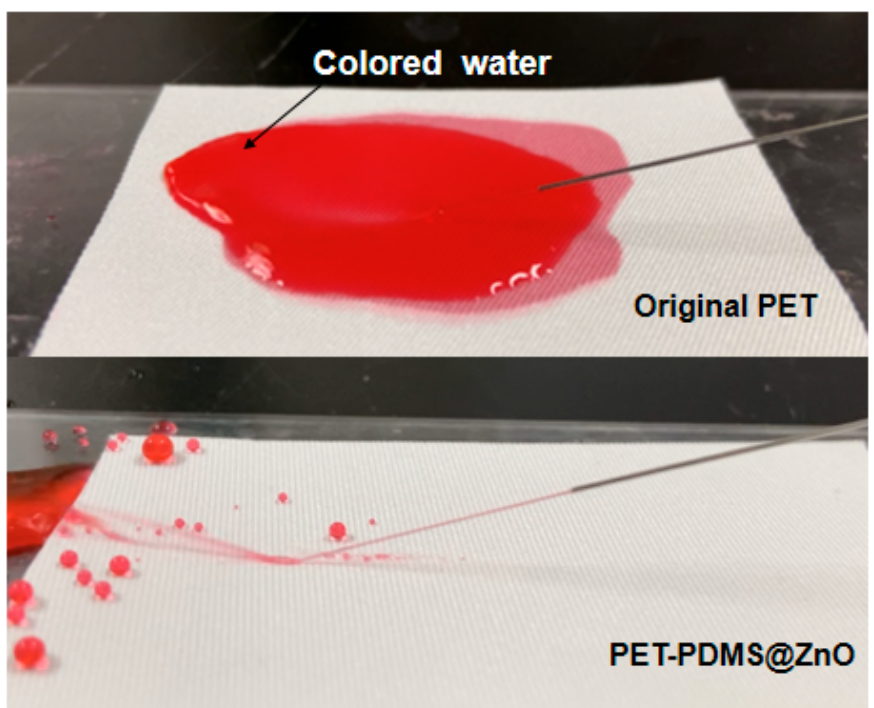

Figure 8. Water jet experiment on pristine PET and PDMS@ZnO fabric, showing good anti-spatter property of PDMS@ZnO fabric.

Since the PET-PDMS@ZnO fabric we prepared has properties of antibacterial and antibacterial adhesion, the dual synergistic among them makes the fabric own excellent antibacterial properties. Moreover, due to the great improvement of hydrophobicity of the PET-PDMS@ZnO fabric (see Figure 1a,c), the hydrostatic permeability of the PETPDMS@ZnO fabric increased from 0.43 KPa to $3.41 \mathrm{KPa}$ (see Table 1), which was a huge 
promotion. With the addition of $\mathrm{ZnO}$ nanoparticles, the surface roughness between fibers was greatly increased, which made the fiber assembly not easy to slip off under the action of external force. That was the reason why breaking strength permeability of PET-PDMS@ZnO fabric got a promotion of $9.0 \%$ compared with the pristine PET fabric. Comparing with pristine PET fabric, the air permeability and moisture permeability of the PET-PDMS@ZnO fabric were slightly decreased. It was due to the fact that PDMS encapsulated $\mathrm{ZnO}$ nanoparticles and adhered to the surface of the fiber, which reduced the porosity of the fabric, thus affecting the passing rate of gas and water molecules. However, the permeability of PET-PDMS@ZnO fabric was basically in line with the standards of medical protective clothing [28]. Furthermore, the thickness of coated fabrics will affect the feeling of wearing, but our test results show that the weight gain rate of the PETPDMS@ZnO fabric is 3.5\%, and the thickness of fabric increased from $0.398 \mathrm{~mm}$ to $0.408 \mathrm{~mm}$, which is basically unchanged. That means protective clothings made of this fabric can retain the advantage of being comfortable. Therefore, it can be applied to protecting clothing or textiles that need to have good antibacterial properties (such as bed sheets, duvet covers, etc.).

Table 1. The wearability of PET-PDMS@ZnO fabrics.

\begin{tabular}{ccc}
\hline Permeabilities & Pristine PET Fabric & PET-PDMS@ZnO Fabric \\
\hline Air permeability $(\mathrm{mm} / \mathrm{s})$ & 15.97 & 13.96 \\
Water-vapor transmission $\left(\mathrm{g} /\left(\mathrm{m}^{2} \cdot \mathrm{d}\right)\right)$ & 8726.40 & 8581.15 \\
Hydrostatic $(\mathrm{KPa})$ & 0.43 & 3.41 \\
Breaking strength $(\mathrm{N})$ & 630.40 & 686.90 \\
\hline
\end{tabular}

\section{Conclusions}

In this study, plasma technology is used to prepare superhydrophobic antibacterial PET fabrics (The PET-PDMS@ZnO fabric). Compared with other methods, plasma technology is simple, clean, friendly, and generates no toxic waste, and the coating monomer of polydimethylsiloxane (PDMS) used is non-toxic, harmless, fluorine-free, and does no harm to the environment. The PET-PDMS@ZnO fabric is superhydrophobic, with a WCA of $162.7^{\circ}$, and SA of $7.5^{\circ}$. What's more, it demonstrates a good water repellency and has good durability; even after 300 washing cycles and 600 friction cycles, it can still keep superhydrophobic property. Here, SEM, EDX, and FTIR were used to confirm the results that the PDMS@ZnO coating was successfully constructed on the fiber surface. Also, the result of FTIR indicates the existence of long chain alkyl groups and Si-O-Si network structure. In addition, the PET-PDMS@ZnO fabric shows good propertities of antibacterial and antibacterial adhesion, and the antibacterial rates of Escherichia coli and Staphylococcus aureus before washing were $99.89 \%$ and $99.85 \%$, respectively, and after 100 cycles of washing, the antibacterial rates decreased to $99.36 \%$ and $99.17 \%$, respectively. What's more, it shows good synergistic properties of antibacterial and antibacterial adhesion, which has huge development prospects in the application of protective clothing and textiles requiring good antibacterial properties (such as bed sheets, duvet covers, etc.).

Author Contributions: Y.L.: Conceptualization, Methodology, Formal analysis, Investigation, Writing-original draft; Y.G.: Supervision, Conceptualization, Investigation, Writing-review \& editing; L.X.: Investigation, Writing-review; X.C.: Investigation, Writing-review; X.Z.: Investigation; G.X.: Investigation, Writing-review; J.S.: Investigation. All authors have read and agreed to the published version of the manuscript.

Funding: This research was funded by the Natural Science Foundation of China (Grant Nos. 11875104, 11475043 and 11705115).

Institutional Review Board Statement: "Not applicable" for studies not involving humans or animals.

Informed Consent Statement: "Not applicable" for studies not involving humans. 
Data Availability Statement: Data is contained within the article.

Acknowledgments: The authors would like to thank Qianhan Han of the College of Science, Donghua University for his guidance on chart making and valuable comments throughout this study.

Conflicts of Interest: The authors declare no conflict of interest.

\section{References}

1. Xie, L.Y.; Hong, F.; Liu, J.H.; Zhang, G.Z.; Wu, C. Intergrated design and study of marine antifouling polymer materials. Acta Polym. Sin. 2012, 1, 1-13. [CrossRef]

2. Crick, C.R.; Ismail, S.; Pratten, J.; Parkin, I.P. An investigation into bacterial attachment to an elastomeric superhydrophobic surface prepared via aerosol assisted deposition. Thin Solid Film. 2011, 519, 3722-3727. [CrossRef]

3. Cano, S.; Carril, M. Recent Developments in the design of non-biofouling coatings for nanoparticle and surfaces. Int. J. Mol. Sci. 2020, 21, 1007. [CrossRef] [PubMed]

4. Stallard, C.P.; McDonnell, K.A.; Onayemi, O.D.; OYPERL, J.P.; Dowling, D.P. Evaluation of protein adsorption on atmospheric plasma seposited coatings exhibiting superhydrophilic to superhydrophobic properties. Biointerphases 2012, 7, 31. [CrossRef] [PubMed]

5. Nguyen-Tri, P.; Altiparmak, F.; Nguyen, N.; Tuduri, L.; Ouellet-Plamondon, C.M.; Prud'homme, R.E. Robust superhydrophobic cotton fibers prepared by simple dip- coating approach using chemical and plasma-etching pretreatments. Acs Omega. 2019, 4, 7829-7837. [CrossRef]

6. Ishizaki, T.; Saito, N.; Takai, O. Correlation of cell adhesive behaviors on superhydrophobic superhydrophilic, and micropatterned superhydrophobic/superhydrophilic surfaces to their surface chemistry. Langmuir 2010, 26, 8147-8154. [CrossRef]

7. Bao, Q.; Nishimura, N.; Kamata, H.; Furue, K.; Terada, A. Antibacterial and anti-biofilm efficacy of fluoropolymer coating by a 2,3,5,6-tetrafluoro-p-phenylenedimethanol structure. Colloid. Surf. B 2016, 151, 363. [CrossRef]

8. Ellinas, K.; Kefallinou, D.; Stamatakis, K.; Gogolides, E.; Tserepi, A. Is there a threshold in the antibacterial action of superhydrophobic surfaces? ACS Appl. Mater. Interfaces 2017, 9, 39781-39789. [CrossRef]

9. Liu, T.; Yin, B.; He, T.; Guo, N.; Dong, L.; Yin, Y. Complementary effects of nanosilver and superhydrophobic coatings on the prevention of marine bacterial adhesion. ACS Appl. Mater. Interfaces 2012, 4, 4683. [CrossRef]

10. Tian, X.; Verho, T.; Ras, R. Moving superhydrophobic surfaces toward real-world applications. Science 2016, 352, 142. [CrossRef]

11. Wu, M.; Ma, B.; Pan, T.; Chen, S.; Sun, J. Silver-nanoparticle-colored cotton fabrics with tunable colors and durable antibacterial and self-healing superhydrophobic properties. Adv. Funct. Mater. 2016, 26, 569-576. [CrossRef]

12. Xu, C.; Wang, Y. One step approach to the growth of $\mathrm{ZnO}$ nano-/microrods on cellulose toward its durable superhydrophobicity. Adv. Mater. Interfaces 2017, 4, 1700550. [CrossRef]

13. Shao, L.; Liu, H.; Zeng, W.P.; Zhou, C.Y.; Dan, L. Immobilized and photocatalytic performances of $\mathrm{PDMS}^{-S_{i O}} \mathrm{C}_{2}-\mathrm{chitosan} @ \mathrm{TiO}_{2}$ composites on pumice under simulated sunlight irradiation. Appl. Surf. Sci. 2019, 478, 1017-1026. [CrossRef]

14. Wang, M.K.; Zhang, Z.Z.; Wang, Y.L.; Zhao, X.; Yang, M.M.; Men, X.H. Superwetting fabrics towards multifunctional applications: Oil/water separation, anti-fouling and flame-retardance. Appl. Surf. Sci. 2020, 508, 145265. [CrossRef]

15. Foorginezhad, S.; Zerafat, M.M. Fabrication of stable fluorine-free superhydrophobic fabrics for anti- adhesion and self-cleaning properties. Appl. Surf. Sci. 2019, 464, 458-471. [CrossRef]

16. Cheng, D.S.; He, M.T.; Li, W.B.; Wu, J.H.; Ran, J.H.; Cai, G.M.; Wang, X. Hydrothermal growing of cluster-like ZnO nanoparticles without crystal seeding on PET films via dopamine anchor. Appl. Surf. Sci. 2019, 467, 534-542. [CrossRef]

17. Lin, D.M.; Zeng, X.R.; Li, H.Q.; Lai, X.J. Facile fabrication of superhydrophobic and flame-retardant coatings on cotton fabrics via layer-by-layer assembly. Cellulose 2018, 25, 3135-3149. [CrossRef]

18. Xu, L.Y.; Guo, Y.; Bai, G.H. Fluorine-free and wearing comfortable superhydrophobic fabrics from particle-free polymer coatings. Prog. Org. Coat. 2020, 146, 105727. [CrossRef]

19. Xu, L.Y.; Deng, J.W.; Guo, Y.; Wang, W.; Zhang, R.Y.; Yu, J.Y. Fabrication of superhydrophobic cotton fabric by low-pressure plasma-enhanced chemical vapor deposition. Text. Res. J. 2019, 89, 1853-1862. [CrossRef]

20. Sun, F.J.; Chen, L.; Sun, W.G. Study on microwave method extraction mugwort pigment and antibacterial performance. Wool Text. J. 2011, 39, 17-19.

21. Tian, Y.; Liu, X.; Zheng, X.; Wang, L. Antimicrobial properties of flax fibers in the enzyme retting process. Fibres Text. East. Eur. 2016, 24, 15-17. [CrossRef]

22. Zhang, J.G.; Xu, Z.W.; Mai, W.; Min, C.Y.; Zhou, B.M.; Shan, M.J.; Li, Y.L.; Yang, C.Y.; Wang, Z.; Qian, X.M. Improved hydrophilicity, permeability, antifouling and mechanical performance of PVDF composite ultrafiltration membranes tailored by oxidized low-dimensional carbon nanomaterials. J. Mater. Chem. A 2013, 1, 3101. [CrossRef]

23. Rau, C.; Kulisch, W. Mechanisms of plasma polymerization of various silico-organic monomer. Thin Solid Film. 1994, 249, 28-37. [CrossRef]

24. Yazdanshenas, M.; Shateri-Khalilabad, M. One-Step synthesis of superhydrophobic coating on cotton fabric by ultrasound irradiation. Ind. Eng. Chem. Res. 2013, 52, 12846-12854. [CrossRef]

25. Agarwal, S.; Nekouei, F.; Kargarzadeh, H. Preparation of Nickel hydroxide nanoplates modified activated carbon for Malachite Green removal from solutions: Kinetic, thermodynamic, isotherm and antibacterial studies. Process. Saf. Environ. 2016, $102,85-97$. 
26. Sirelkhatim, A.; Mahmud, S.; Seeni, A. Review on Zinc Oxide Nanoparticles: Antibacterial activity and toxicity mechanism. Nano-Micro Letters. 2015, 7, 219-242. [CrossRef]

27. Seth, M.; Khan, H.; Jana, S. Hierarchically structured alpha-nickel hydroxide based superhydrophobic and antibacterial coating on cellulosic materials for oil-water separation. Mater. Chem. Phys. 2020, 249, 123030. [CrossRef]

28. Li, Y.; Cai, R.; Lu, Y. Selection and use of protective clothing in novel coronavirus pneumonia epidemic. Chin. J. Infect. Cont. 2020, $19,117-122$. 